\title{
Estudo das lesões de sobrecarga durante período intenso de atividade física complementado com avaliação por dinamometria isocinética
}

\section{Study on Overload Injuries during Periods of Intense Physical Activity Complemented by Isokinetic Dynamometry Evaluation}

\author{
José Lito Mónico ${ }^{1}$ Frederico Silva ${ }^{1}$ José Pedro Marques ${ }^{1}$ Joaquim Cardoso ${ }^{1}$ Lisete Mónico ${ }^{2}$
}

Fernando Fonseca ${ }^{3}$

${ }^{1}$ Centro de Saúde Militar de Coimbra, Coimbra, Portugal

2 Faculdade de Psicologia e de Ciências da Educação da Universidade de Coimbra, Coimbra, Portugal

3 Serviço de Ortopedia e Traumatologia do Centro Hospitalar e

Universitário de Coimbra, Coimbra, Portugal
Endereço para correspondência José Lito Mónico, MSc, Centro de Saúde Militar de Coimbra, R. Vandelli, 2, 3030-405, Coimbra, Portugal (e-mail: jlmonico@gmail.com).

Rev Bras Ortop 2020;55(6):681-686.

\section{Resumo}

Palavras-chave

- lesões de sobrecarga

- dinamometria isocinética

- esforço físico

- militares
Objetivo Os autores pretendem medir a incidência de lesões de sobrecarga em militares em formação, que são submetidos a exercício físico intenso, e compará-la com um grupo controle. Posteriormente, pretende-se verificar se existe alguma relação entre a ocorrência de lesões de sobrecarga e alguns parâmetros da função neuromuscular.

Métodos Estudo observacional prospectivo analítico. Grupo de observação e grupo controle constituídos por militares do Exército Português. A avaliação clínica foi feita por entrevista médica na semana que antecede o início do curso de paraquedismo militar e na semana imediatamente após o final do curso. Em simultâneo com a entrevista médica, foi realizada a avaliação da performance neuromuscular através da dinamometria isocinética.

Resultados Com 44 dos 57 militares em formação a referir queixas álgicas, o grupo de observação apresentou significativamente mais lesões que o grupo controle $(p<0.001)$. Cinco queixas foram de origem traumática e 39 foram lesões de sobrecarga. Dos 39 militares com lesões de sobrecarga, 21 referiram limitação do rendimento esportivo. No entanto, na avaliação por dinamometria isocinética, não se verificaram diferenças estatisticamente significativas na evolução da performance neuromuscular ( $p=0.223$ e $p=0.229$ ).

Conclusão Os militares em formação são indivíduos propensos a sofrerem lesões de sobrecarga, tendo-se obtido uma taxa de incidência de lesões de sobrecarga na ordem dos 70\%. A implementação de estratégias de monitoração e prevenção das lesões são fundamentais na promoção da saúde e da capacidade física.

Trabalho desenvolvido no Centro de Saúde Militar de Coimbra,

Coimbra, Portugal.

recebido

21 de Novembro de 2019

aceito

02 de Março de 2020
DOI https://doi.org/

10.1055/s-0040-1712137. ISSN 0102-3616.
Copyright $\odot 2020$ by Sociedade Brasileira License terms de Ortopedia e Traumatologia. Published by Thieme Revinter Publicações Ltda, Rio de Janeiro, Brazil 


\begin{abstract}
Keywords

- overload injuries

- isokinetic dynamometry

- physical activity

- military personnel

Objective The present study aims to measure the incidence of overload injuries in training soldiers, who are subjected to intense physical exercise, and to compare it with a control group. Next, it intends to verify whether there is any relationship between overload injuries and some neuromuscular function parameters.

Methods Analytical, prospective observational study. Both the observational and the control group consisted of soldiers from the Portuguese Army. Clinical evaluation was carried out by medical interview in the week prior to the beginning of a military parachuting course and in the week immediately after its completion. The neuromuscular performance was assessed by isokinetic dynamometry during the medical interview.

Results With 44 of the 57 military personnel in training complaining of pain, the observational group had significantly more injuries than the control group $(p<0.001)$. Five complaints had traumatic origin and 39 were overload injuries. Of the 39 military personnel with overload injuries, 21 reported limited sports performance. However, isokinetic dynamometry showed no statistically significant differences in neuromuscular performance $(p=0.223$ and $p=0.229)$.

Conclusion Military personnel in training are prone to overload injuries, with an incidence rate $>70 \%$. The implementation of strategies for injury monitoring and prevention is critical to promote health and physical capacity.
\end{abstract}

\section{Introdução}

As lesões de sobrecarga são lesões sem um traumatismo agudo identificável, e têm sido referidas como o tipo de lesão mais frequente na prática esportiva. ${ }^{1,2}$ Estas lesões são provocadas por microtraumatismos de repetição, em um contexto em que a carga aplicada supera a capacidade adaptativa tecidular e/ou há uma recuperação insuficiente entre as aplicações, lesando progressivamente as estruturas atingidas. ${ }^{1-5} \mathrm{~A}$ fisiopatologia envolvida neste tipo de lesões não é ainda completamente compreendida, sendo os seus modelos correntes baseados em conceitos teóricos. ${ }^{4,6} \mathrm{O}$ modelo teórico sobre lesões tendinosas (-Figura 1) foca o equilíbrio entre a carga aplicada e o repouso tecidular como fator preditivo de lesão. ${ }^{4}$ Lesões prévias e preparação física inadequada também são outros fatores associados. ${ }^{7}$ De acordo com outros trabalhos, ${ }^{8,9} 66 \%$ dos atletas que treinam 20 a 35 horas semanais durante um ano desenvolvem lesões de sobrecarga limitantes do desempenho, ${ }^{8}$ sendo uma causa comum de abandono prematuro da atividade esportiva ${ }^{9}$ e de dor crônica. ${ }^{9-11} \mathrm{~A}$ avaliação da função neuromuscular com recurso à dinamometria isocinética é comum, sendo útil no diagnóstico de lesões e disfunções musculosqueléticas. ${ }^{12}$ No contexto militar, os militares são muitas vezes expostos a vários agentes estressantes, como um prolongado e extenuante exercício físico, déficits de hidratação, condições climatéricas adversas e privação de sono. ${ }^{13-15} \mathrm{~A}$ identificação e compreensão destes fatores favorecedores deste tipo de lesões ${ }^{13-15}$ é o primeiro passo para a sua prevenção. ${ }^{16}$

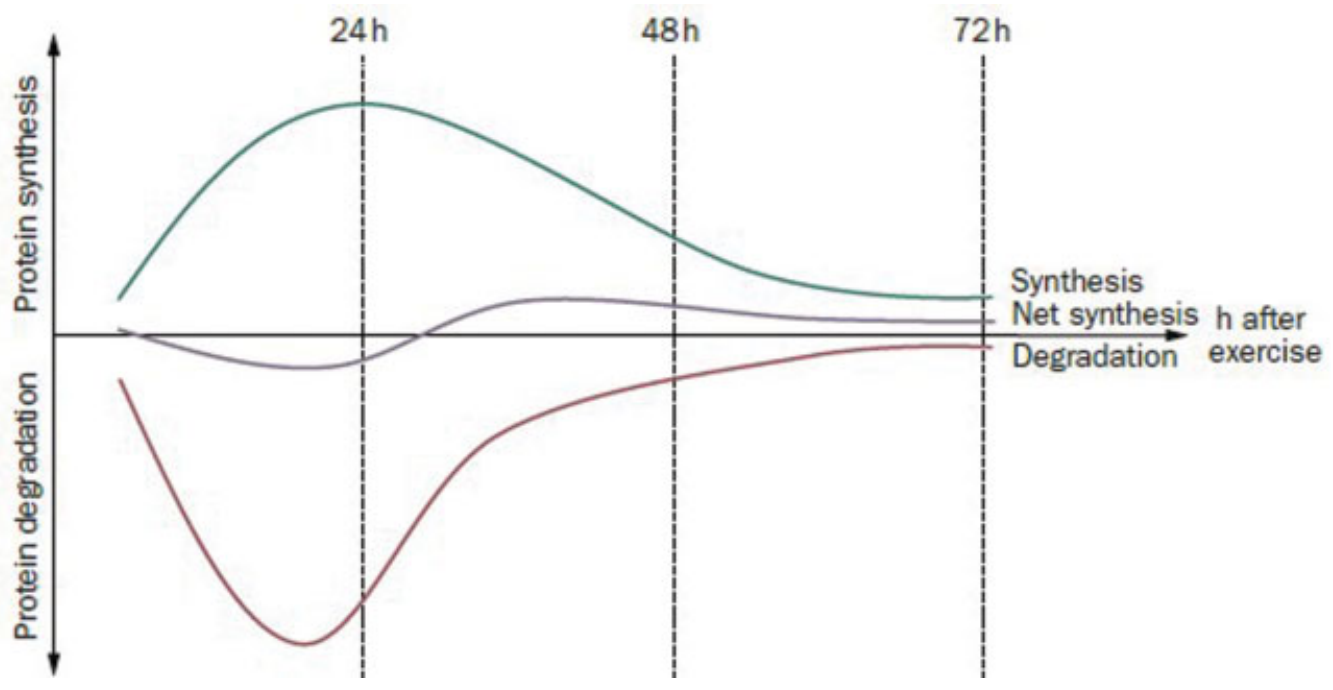

Fig. 1 Representação esquemática da síntese e degradação de colágeno após uma sessão de exercício físico. ${ }^{4}$ 
Os autores pretendem medir a incidência de lesões de sobrecarga em militares em formação, que são submetidos a exercício físico intenso, e compará-la com um grupo controle, constituído por militares que apenas fazem o seu treino físico habitual. Posteriormente, pretende-se verificar se existe alguma relação entre a ocorrência de lesões de sobrecarga e alguns parâmetros da função neuromuscular.

Como objetivos secundários, os autores pretendem: avaliar a evolução da performance neuromuscular e saber se esta é influenciada pela dor.

\section{Método}

\section{Tipo de Estudo, População e Amostra}

Trata-se de um estudo observacional prospectivo analítico. A população-alvo é constituída por militares do Exército Português. A amostra é composta por 2 grupos: 1) grupo de observação, que integra 57 soldados do sexo masculino (idades compreendidas entre 19 e 25 anos, inclusive; $M=21$ anos) que frequentaram o curso de Paraquedismo Militar do Exército Português; 2) grupo de controle, composto por 30 soldados do sexo masculino (idades compreendidas entre 21 e 28 anos, inclusive; $M=25$ anos) que não frequentaram qualquer curso do Exército.

Dos participantes do grupo de observação, foi selecionada uma amostra aleatória constituída por 38 soldados para efeitos de realização de testes de dinamometria isocinética. Como critérios de inclusão para o grupo de observação, foram considerados os militares que concluíram com sucesso o curso de Paraquedismo Militar do Exército Português e realizaram as avaliações médicas previstas. Para o grupo de controle, foram incluídos os militares que realizaram as avaliações médicas previstas e não participaram em qualquer curso do Exército. Como critérios de exclusão no grupo observacional, foram eliminados os militares que abandonaram o curso de Paraquedismo Militar do Exército Português e os que não realizaram as avaliações médicas previstas. No grupo controle, foram excluídos os militares que não realizaram as avaliações médicas previstas.

\section{Materiais e Procedimentos}

O presente estudo realizou-se no Laboratório de Fisiologia do Exercício de um Centro de Saúde Militar. A avaliação clínica através de uma entrevista médica foi realizada em dois momentos: na semana que antecede o início do curso de paraquedismo militar e na semana imediatamente após o final do curso. Na primeira fase, a entrevista clínica realizada consistiu numa anamnese dirigida e na realização do exame objetivo. O principal enfoque da entrevista médica da segunda fase foi a averiguação de queixas musculoesqueléticas existentes durante o curso de paraquedismo, o tratamento médico realizado e a forma como estas queixas afetaram o respectivo rendimento físico.

Para avaliação da dor foi utilizada uma escala de 0 a 10 valores, sendo 0 correspondente ao estado "sem dor" e 10 à "dor máxima”. Para avaliação da alteração do rendimento físico provocado pela dor, foi utilizada uma escala de autoa- valiação de 0 a 2 pontos sendo: 0 classificado como "sem alteração", 1 como "ligeira diminuição do rendimento" e 2 como "limitação moderada a severa do rendimento". No presente trabalho, para efeitos de registro das lesões desportivas, foi usada a definição 'qualquer queixa física' dos consensos F-MARC. ${ }^{17}$

A avaliação isocinética foi realizada em dois momentos, na semana que antecedia o início do curso de paraquedismo e na semana após a conclusão deste. Para a referida avaliação, realizou-se um aquecimento prévio num aparelho de remo, Concept 2' (Concept 2, Inc. 105 Industrial Park Drive Morrisville, Vermont USA), com a duração de 5 minutos. Posteriormente, utilizou-se o dinamômetro HUMAC/NORM Testing and Rehabilitation System com a aplicação do software informático Humac (Computer Sports Medicine Inc. 101 Tosca Drive Stoughton, Massachusetts USA). Realizaram-se 5 repetições de extensão/flexão do joelho no modo concêntrico-concêntrico numa velocidade de $60^{\circ} /$ segundo com uma amplitude de movimentos compreendida entre os $100^{\circ}$ de flexão e os $0^{\circ}$, tempo de repouso de 2 minutos entre testes. Estes procedimentos foram efetuados para ambos os membros inferiores.

\section{Atividades Realizadas pelo Grupo de Observação}

No Curso de Paraquedismo Militar, a formação de praças paraquedistas tem uma duração de 17 semanas: preparação para o Curso de Combate (2 semanas), Curso de Combate (9 semanas), preparação para o Curso de Paraquedismo (2 semanas) e pelo Curso de Paraquedismo (4 semanas). 0 curso de combate consiste num período intenso de instrução de técnica individual de combate e de técnica de combate de secção. Tipicamente, a instrução tem início às 8 h00 e termina às $24 \mathrm{~h} 00$, perfazendo um total de 16 horas de instrução diárias. As atividades físicas são habitualmente realizadas com o uniforme camuflado militar, botas com sola de borracha, capacete e arma (espingarda automática Galil). Em determinadas atividades, os alunos fazem-se acompanhar de uma mochila, transportando cerca de $20 \mathrm{~kg}$ de peso em material. O Curso de Paraquedismo é um período de formação técnica sobre o uso de todos os equipamentos necessários ao salto em paraquedas. Habitualmente, a instrução tem início às 8 h00 e termina às $17 \mathrm{~h} 00$, com duas instruções noturnas por semana. As atividades físicas são realizadas sempre com farda de trabalho (uniforme camuflado e botas).

\section{Atividades Realizadas pelo Grupo de Controle}

No grupo de controlo, os militares realizavam uma hora de treino físico 3 vezes por semana em dias alternados, sendo que o exercício físico realizado e sua respectiva carga definida pelo próprio militar. O equipamento de treino era composto por calções, $t$-shirt e sapatilhas.

\section{Análise dos Dados}

Para análise e tratamento estatístico dos dados, foi utilizado o programa IBM SPSS Statistics for Windows, Version 22.0 (IBM Corp., Armonk, NY, EUA).

Os testes de aderência à normalidade Shapiro-Wilk (S-W) legitimaram a utilização de estatísticas paramétricas, visto que indicaram uma distribuição normal nas variáveis em 
análise: diferenciais do pico de Torque(PT) dos músculos extensores e flexores de ambas as coxas e nos grupos organizados segundo o padrão de dor $(\mathrm{S}-\mathrm{W}<0,99$; $p>0,13)$, PT direito diferencial $(\mathrm{S}-\mathrm{W}=0,96 ; p=0,24)$, PT esquerdo diferencial $(S-W=0,98 ; p=0,67)$, escala da dor (S-W $<0,96 ; p>0,80)$ e diferenciais do PT dos músculos extensores da coxa direitos e esquerdos ( $\mathrm{S}-\mathrm{W}<0,98$; $p>$ $0,59)$. Foram assim utilizados os testes de $X^{2} t$ de Student, análise de variância (ANOVA, na sigla em inglês) e comparações múltiplas Fisher LSD. Considerou-se um erro de tipo I de $p<0,05$ em todas as análises.

\section{Resultados}

Durante o período de estudo, verificamos que, no grupo observacional, 44 militares referiram queixas álgicas durante a frequência do curso de paraquedismo versus 13 militares que negaram a sensação de dor. No grupo controle, apenas três militares referiram lombalgia. Verificou-se que os participantes do grupo de observação apresentaram significativamente mais lesões durante o período avaliado comparativamente ao grupo controle, $X^{2}(1)=47,86 ; p<0,001$. Dos 44 militares queixosos do grupo observacional, cinco referiram dor após um traumatismo, enquanto 39 militares não tinham um traumatismo identificado. Da análise às lesões de sobrecarga obtidas em ambos os grupos, verificou-se que os participantes do grupo de observação apresentaram significativamente mais lesões de sobrecarga durante o período avaliado comparativamente ao grupo controle, $X^{2}(1)=37,20 ; p<0.001$. Nos militares com lesões de sobrecarga, constatou-se que o joelho foi a principal região afetada ( - Tabela $\mathbf{1}$ ) e que a corrida, a marcha e a instrução militar foram as principais atividades responsáveis pelas queixas (-Tabela 2). Quanto ao rendimento físico desses militares, 21 referiram limitações no rendimento causadas pelas queixas álgicas, enquanto 18 negaram a existência de qualquer limitação.

No que diz respeito às modalidades de tratamento instituídas, constatou-se que todos os militares referiram melhoria das queixas com o repouso e a medicação com anti-inflamatórios não esteroides ( - Tabela 3 ). Com o término do curso, $39 \%$ dos militares queixosos ainda mantinham o mesmo quadro álgico. Foram estabelecidos vários diagnósticos presuntivos, com destaque para a síndrome femoropatelar que afetou cerca de $60 \%$ dos militares queixosos (-Tabela 4 ).

Tabela 1 Incidência da dor distribuída por regiões anatômicas

\begin{tabular}{|l|l|l|}
\hline & $\mathbf{n}$ & $\%$ \\
\hline Ombro e braço & 2 & 5.1 \\
\hline Joelho & 27 & 69.2 \\
\hline Perna & 1 & 2.6 \\
\hline Tendão de aquiles & 3 & 7.7 \\
\hline Pé & 3 & 7.7 \\
\hline Coluna lombar & 3 & 7.7 \\
\hline Total & 39 & 100.0 \\
\hline
\end{tabular}

Tabela 2 Atividade desenvolvida quando iniciou da dor

\begin{tabular}{|l|l|l|}
\hline & Frequência & $\%$ \\
\hline Marcha e instrução militar & 29 & 74,4 \\
\hline Corrida & 8 & 20,5 \\
\hline Pista de cordas & 2 & 5.1 \\
\hline Total & 39 & 100 \\
\hline
\end{tabular}

Tabela 3 Modalidades de tratamento observadas

\begin{tabular}{|l|l|l|}
\hline & $\mathbf{n}$ & $\%$ \\
\hline Repouso & 4 & 10.3 \\
\hline Repouso e anti-Inflamatórios & 33 & 84.6 \\
\hline Repouso, anti-Inflamatórios e fisioterapia & 2 & 5.1 \\
\hline Total & 39 & 100.0 \\
\hline
\end{tabular}

Nas avaliações da performance neuromuscular (- Tabela 5), verificou-se um aumento do PT médio. Para apurar as diferenças entre a evolução da performance neuromuscular e a dor apresentada, os soldados do grupo de observação foram subdivididos em 3 grupos, correspondendo a três níveis da variável independente (VI): 1) sem dor durante o curso $(18,4 \%) ; 2)$ com dor apenas durante o curso (39,5\%); e 3 ) com dor durante e após o final do curso (42,1\%). Não se verificaram diferenças estatisticamente significativas entre estes três grupos, tanto para a variável dependente (VD) PT direito diferencial (PT final - PT inicial), $F(2,31)=1,01$; $p=0,377$, quanto para a VD PT esquerdo diferencial, $F$ $(2,31)=1,21, p=0,313$. Os testes de comparação múltipla de Fisher LSD também não identificaram qualquer diferença estatisticamente significativa.

Analisando a evolução da performance neuromuscular com a autopercepção do rendimento físico, não se verificaram diferenças estatisticamente significativas, PT diferencial direito, $t(27)=1,25 ; p=0.223$, e esquerdo, $t(27)=1.23 ; p=0.229$.

\section{Discussão}

A corrida é uma das atividades desportivas mais populares, não obstante dos reconhecidos benefícios para a saúde associados a esta atividade, ela está implicada numa série de patologias de

Tabela 4 Diagnósticos presuntivos estabelecidos

\begin{tabular}{|l|l|l|}
\hline & $\mathbf{n}$ & $\%$ \\
\hline Estresse patelofemoral & 24 & 61.5 \\
\hline Síndrome banda iliotibial & 1 & 2.6 \\
\hline Tendinite de aquiles & 3 & 7.7 \\
\hline Fasceíte plantar & 2 & 5.1 \\
\hline Tendinite não especificada & 6 & 15.4 \\
\hline Lombalgia & 3 & 7.7 \\
\hline Total & 39 & 100.0 \\
\hline
\end{tabular}


Tabela 5 Valores das avaliações de dinamometria isocinética

\begin{tabular}{|c|c|c|c|}
\hline & Mínimo & Máximo & Média \\
\hline $60^{\circ} /$ segundo extensores PT Direito $1^{\text {a }}$ Avaliação & 129 & 247 & 183.55 \\
\hline $60^{\circ} /$ segundo extensores PT Direito $2^{\text {a }}$ Avaliação & 114 & 252 & 190.39 \\
\hline $60^{\circ} /$ segundo extensores PT Direito Diferencial & -13 & 33 & 3.95 \\
\hline $60^{\circ} /$ segundo flexores PT Direito $1^{a}$ Avaliação & 72 & 172 & 123.32 \\
\hline $60^{\circ} /$ segundo flexores PT Direito $2^{a}$ Avaliação & 76 & 186 & 135.24 \\
\hline $60^{\circ} /$ segundo flexores PT Direito Diferencial & -22 & 52 & 9.92 \\
\hline $60^{\circ} /$ segundo extensores PT Esquerdo $1^{a}$ Avaliação & 104 & 259 & 173.18 \\
\hline $60^{\circ} /$ segundo extensores PT Esquerdo 2a Avaliação & 115 & 270 & 185.79 \\
\hline $60^{\circ} /$ segundo extensores PT Esquerdo Diferencial & -18 & 28 & 7.76 \\
\hline $60^{\circ} /$ segundo flexores PT Esquerdo 1a Avaliação & 69 & 160 & 118.79 \\
\hline $60^{\circ} /$ segundo flexores PT Esquerdo $2^{\text {a }}$ Avaliação & 81 & 183 & 132.21 \\
\hline $60^{\circ} /$ segundo flexores PT Esquerdo Diferencial & -33 & 35 & 11.76 \\
\hline Proporção flexores/extensores Direito 1a Avaliação & 50 & 88 & 68.29 \\
\hline Proporção flexores/extensores Direito $2^{a}$ Avaliação & 48 & 96 & 71.97 \\
\hline Proporção flexores/extensores Esquerdo 1a Avaliação & 53 & 84 & 68.87 \\
\hline Proporção flexores/extensores Esquerdo 2a Avaliação & 55 & 86 & 70.76 \\
\hline
\end{tabular}

sobrecarga, predominantemente no membro inferior. ${ }^{7,18}$ Nesta região anatômica, as patologias mais frequentemente observadas são: síndrome femoropatelar, tendinopatia aquiliana, tendinopatia rotuliana, síndrome de estresse medial da tíbia, fasceíte plantar e fraturas de stress. ${ }^{19}$ É de salientar que a esmagadora maioria das queixas teve início durante a corrida, marcha ou instrução militar, que são atividades extremamente exigentes para os membros inferiores. Deve-se referir ainda que $\sim 80 \%$ das queixas dos participantes tiveram início durante o curso de combate, facto que pode ser explicado pelo uso constante de botas com sola de borracha, que têm capacidade limitada de absorção do choque, a elevada exigência desta fase, os períodos insuficientes de repouso e ao uso de fardamento/ equipamento pesado. Os modelos teóricos de lesões tendinosas 4
(-Figura 1) e das forças de reação ao $\operatorname{solo}^{19}$ (-Figura 2) corroboram estes fatos e funcionam como modelos preditores de lesões de sobrecarga.

Todos os militares referiram melhoria das queixas com o repouso durante os períodos de fim de semana. No entanto, apenas cerca de $10 \%$ dos alunos referiram remissão dos sintomas sem o recurso a qualquer outro tipo de tratamento. Salienta-se a elevada percentagem de soldados a recorrerem empiricamente a anti-inflamatórios não esteroides (84,6\%), enquanto apenas dois militares $(5,0 \%)$ recorreram à reabilitação com fisioterapia. Efetivamente, a modificação da atividade física é o ponto basilar no tratamento médico de qualquer lesão de sobrecarga. Outras modalidades de tratamento eficazes prendem-se com a aplicação tópica de gelo, administração

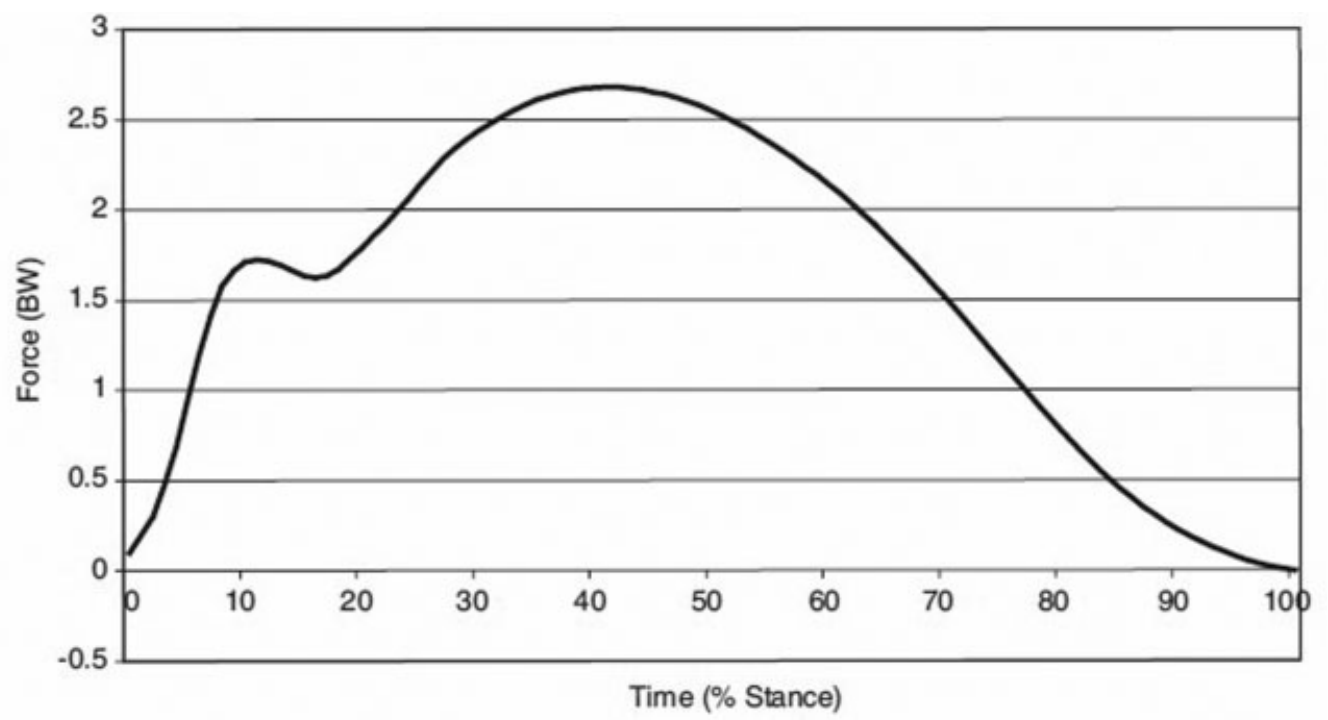

Fig. 2 Esquema representativo da componente vertical das forças de reação ao solo durante o ciclo de marcha. ${ }^{19}$ 
de anti-inflamatório não esteroide oral e a realização de exercícios de reabilitação. ${ }^{20}$ Contudo, durante a frequência do curso de paraquedismo, os soldados alunos têm de acompanhar a instrução e corresponder da melhor forma possível à carga física imposta sob pena de exclusão do curso, sendo este um potencial fator de agravamento das lesões estabelecidas. Deve-se referir que a reabilitação com fisioterapia, não disponível na unidade, foi feita apenas durante o fim de semana e a título particular pelos soldados alunos interessados, justificando a sua baixa taxa de realização. A crioterapia é um tratamento barato e de fácil administração que aumenta o limiar da dor, aumenta a capacidade de deformação plástica dos tecidos e está associada à inibição da cascata da inflamação. ${ }^{21}$ No entanto, não se verificou o uso de gelo tópico no tratamento das lesões apresentadas, em parte justificado pela sua reduzida acessibilidade durante a frequência do curso.

No presente estudo, verificou-se um aumento do PT médio, especialmente nos músculos inicialmente mais fracos que registraram uma maior progressão, que resultou numa redução das assimetrias musculares. Um resultado esperável tendo em conta que os exercícios realizados durante o período de formação estimulam ambos os membros da mesma maneira.

Uma otimização da carga aplicada é um pré-requisito básico para o bom desenvolvimento da performance neuromuscular. ${ }^{22}$ Neste estudo, não se verificou qualquer diferença estatisticamente significativa entre a performance dos militares que nunca referiram dor e os militares lesionados. Avaliando os militares que referiram limitação desportiva associada a um quadro álgico, também não se verifica qualquer diferença estatisticamente significativa comparativamente aos militares que negaram esta limitação. Esta padronização de resultados inesperada pode ser explicada por vários fatores: a curta duração do curso que não permite uma diferenciação maior entre o rendimento dos militares; a carga física semelhante para todos os alunos; e porque a frequência destes cursos tem um caráter eliminatório para quem não revela uma aptidão e desempenho físico adequado à carga imposta.

\section{Conclusão}

As lesões de sobrecarga englobam um variado conjunto de patologias bastante distintas entre si e estão intimamente relacionadas à prática de exercício físico intenso. $\mathrm{O}$ risco de desenvolvimento deste tipo de lesões está associado tanto a fatores intrínsecos ao atleta como a fatores extrínsecos. Não existindo controle sobre a carga física realizada, o equipamento utilizado ou as condições ambientais durante a realização do exercício físico, os militares em formação são, por excelência, indivíduos propensos a sofrerem lesões de sobrecarga, tendo-se obtido um taxa de incidência de lesões de sobrecarga na ordem dos $70 \%$.

A evolução da performance neuromuscular entre os soldados que frequentaram o curso de paraquedismo até o fim foi semelhante.

A implementação de estratégias de monitoração e prevenção destas lesões são fundamentais na promoção da saúde e da capacidade física do atleta. Das várias medidas a adotar, salienta-se: a diminuição da carga física do curso de combate, o aligeiramento do equipamento militar e a melhoria do calçado.

Conflito de Interesses

Os autores declaram não haver conflito de interesses.

\section{Referências}

1 Clarsen B. Overuse Injuries in Sport [dissertation]. Oslo, Norway: Norwegian School of Sport Sciences; 2015

2 Hreljac A. Impact and overuse injuries in runners. Med Sci Sports Exerc 2004;36(05):845-849

3 DiFiori JP, Benjamin HJ, Brenner J, et al. Overuse injuries and burnout in youth sports: a position statement from the American Medical Society for Sports Medicine. Clin J Sport Med 2014;24(01):3-20

4 Magnusson SP, Langberg $\mathrm{H}$, Kjaer M. The pathogenesis of tendinopathy: balancing the response to loading. Nat Rev Rheumatol 2010;6(05):262-268

5 van Wilgen $\mathrm{CP}$, Verhagen EA. A qualitative study on overuse injuries: the beliefs of athletes and coaches. J Sci Med Sport 2012;15(02):116-121

6 Warden SJ, Davis IS, Fredericson M. Management and prevention of bone stress injuries in long-distance runners. J Orthop Sports Phys Ther 2014;44(10):749-765

7 van Mechelen W. Running injuries. A review of the epidemiological literature. Sports Med 1992;14(05):320-335

8 Jacobsson J, Timpka T, Kowalski J, et al. Injury patterns in Swedish elite athletics: annual incidence, injury types and risk factors. Br J Sports Med 2013;47(15):941-952

9 Cook JL, Khan KM, Harcourt PR, Grant M, Young DA, Bonar SF; The Victorian Institute of Sport Tendon Study Group. A cross sectional study of 100 athletes with jumper's knee managed conservatively and surgically. Br J Sports Med 1997;31(04):332-336

10 Jonas S, Phillips EM. ACSM's Exercise is Medicine: A Clinician's Guide to Exercise Prescription. Philadelphia: Wolters Kluwer; 2009

11 Miller M, Thompson S. Delee \& Drez Orthopaedic Sports Medicine. 4th ed. Philadelphia: Saunders/Elsevier; 2015

12 Dvir Z. Isokinetics: Muscle Testing, Interpretation and Clinical Applications. 2nd ed. New York: Churchill Livingstone; 2003

13 Nindl BC, Leone CD, Tharion WJ, et al. Physical performance responses during $72 \mathrm{~h}$ of military operational stress. Med Sci Sports Exerc 2002;34(11):1814-1822

14 Nindl BC, Barnes BR, Alemany JA, Frykman PN, Shippee RL, Friedl KE. Physiological consequences of U.S. Army Ranger training. Med Sci Sports Exerc 2007;39(08):1380-1387

15 Opstad PK, Wiik P, Haugen AH, Skrede KK. Medical consequences in young men of prolonged physical stress with sleep and energy deficiency. NDRE/Publication 95/05586. Norwegian Defence Research Establishment; 1995

16 Välimäki VV, Alfthan H, Lehmuskallio E, et al. Risk factors for clinical stress fractures in male military recruits: a prospective cohort study. Bone 2005;37(02):267-273

17 Dvorak J, Junge A, Grimm K, Eds. F-MARC Football Medicine Manual. 2nd ed. FIFA-Strasse Switzerland; 2009

18 Schwellnus MP, Jordaan G, Noakes TD. Prevention of common overuse injuries by the use of shock absorbing insoles. A prospective study. Am J Sports Med 1990;18(06):636-641

19 Hreljac A. Etiology, prevention, and early intervention of overuse injuries in runners: a biomechanical perspective. Phys Med Rehabil Clin N Am 2005;16(03):651-667, vivi

20 Hess GP, Cappiello WL, Poole RM, Hunter SC. Prevention and treatment of overuse tendon injuries. Sports Med 1989;8(06):371-384

21 Swenson C, Swärd L, Karlsson J. Cryotherapy in sports medicine. Scand J Med Sci Sports 1996;6(04):193-200

22 Kawamori N, Haff GG. The optimal training load for the development of muscular power.J Strength Cond Res 2004;18(03):675-684 